\title{
Fiber-integrated Brillouin microspectroscopy: Towards Brillouin endoscopy
}

\author{
Irina V. Kabakova*, YuChen Xiang, Carl Paterson and Peter Török \\ Blackett Laboratory, Imperial College London \\ Prince Consort Road SW7 2AZ, London, UK \\ *i.kabakova@imperial.ac.uk
}

Received 30 June 2017

Accepted 18 August 2017

Published 13 September 2017

\begin{abstract}
Brillouin imaging (BI) for micromechanical characterization of tissues and biomaterials is a fast-developing field of research with a strong potential for medical diagnosis of disease-modified tissues and cells. Although the principles of BI imply its compatibility with in vivo and in situ measurements, the integration of BI with a flexible catheter, capable of reaching the region of interest within the body, is yet to be reported. Here, for the first time, we experimentally investigate integration of the Brillouin spectroscope with standard optical fiber components to achieve a Brillouin endoscope. The performance of single-fiber and dual-fiber endoscopes are demonstrated and analyzed. We show that a major challenge in construction of Brillouin endoscopes is the strong backward Brillouin scattering in the optical fiber and we present a dualfiber geometry as a possible solution. Measurements of Brillouin spectra in test liquids (water, ethanol and glycerol) are demonstrated using the dual-fiber endoscope and its performance is analyzed numerically with the help of a beam propagation model.
\end{abstract}

Keywords: Brillouin imaging; fiber integration; endoscope.

\section{Introduction}

Micromechanical properties of tissues, cells and biomaterials present key information for understanding their physiological function and for evaluation of pathologic conditions. Brillouin imaging (BI) is an optical technique for mapping elasticity in three-dimensions with micron resolution, ${ }^{1}$ and as such it can be used to assess micromechanical properties of biomaterials. BI is based on the principle of inelastic scattering of light by thermally activated acoustic vibrations or phonons. ${ }^{2}$ Since the technique uses a light beam of relatively low power and requires no physical contact with the tissue, the measurement is thought to be noninvasive and suitable for in vivo diagnosis. ${ }^{3-5}$

Application of Brillouin spectroscopy to biology and biomedicine was suggested in $1966,{ }^{6}$ but the first experimental observation of Brillouin spectra

*Corresponding author.

This is an Open Access article published by World Scientific Publishing Company. It is distributed under the terms of the Creative Commons Attribution 4.0 (CC-BY) License. Further distribution of this work is permitted, provided the original work is properly cited. 
in a biologically-relevant material, gelatin gels, was achieved in $1976 .{ }^{7}$ Shortly after, elastic constants were determined for collagen fibers and muscle tissues. $^{8-10}$ In early work, Brillouin spectra were acquired using multi-stage scanning Fabry-Perot interferometers,${ }^{11}$ since high sensitivity and megahertz resolution are required for isolation of the Stokes and anti-Stokes (AS) signals from the unshifted elastically scattered light. Although tandem scanning Fabry-Perot interferometers yield spectra with high signal-to-noise ratios, the measurement at a single point inside the sample typically takes long time, from minutes to hours, restricting the applicability of the method for point measurements only.

In 2005, Koski and Yarger introduced a new spectrometer-based on a tilted Fabry-Perot etalon. ${ }^{1}$ The angle-dispersive spectrometer simplified the system significantly and allowed to accomplish Brillouin measurements at faster rates, compared to scanning Fabry-Perot interferometers. An addition of high-reflectivity coatings and a transmission window on the surface of the etalon improved the power efficiency of the spectrometer and resulted in a new instrument, so-called virtual-image phasearray (VIPA). ${ }^{12}$ Use of VIPA spectrometers makes it possible to acquire a three-dimensional map of object's elasticity in just a few minutes, a timescale better suited for biomedical imaging. ${ }^{3}$

The main technological developments in the field of BI to date have concentrated on improvements in the spectral resolution, the sensitivity or the acquisition time of the spectrometers used for isolation of Brillouin signals. ${ }^{14-17}$ The sample-facing part of the optical setup was kept unchanged, heavily relying on bulk optical elements or being integrated with a confocal microscopy system. ${ }^{3}$ Thus, so far Brillouin microscopes and spectroscopes are mostly confined to research laboratory environment, with the system footprint requirement of a full optical table and constant adjustment of the optical alignment. To realize the technique's potential for in vivo and in situ biomedical imaging, for which Brillouin signals need to be measured at a given location inside the body calls for system integration with flexible and miniature probes to replace bulk optics and reduce the system complexity and footprint. Fiber-optical probes compatible with standard size flexible endoscopes (1-2 mm) could be an ideal solution for achieving scalable and portable Brillouin imaging systems.
In this paper, we discuss the route taken towards integration of the Brillouin spectroscope with a fiber-optical probe for the laser light delivery and the scattered signal collection. We present, for the first time to our knowledge Brillouin scattering measurements obtained by means of a fiber-optical probe. We demonstrate Brillouin microspectroscopy measurement using two types of probe, based on single- and dual-fiber geometries. We find that construction of a fiber probe for Brillouin endoscopy presents similar challenges to that of Raman endoscopy. ${ }^{18}$ Brillouin scattering from inside the optical fiber results in a strong signal that swamps that from the sample under test. We show that this can be overcome by using a dual fiber probe which uses separate fibers for delivery and collection of the scattered signal. The efficiency of the scattered light collection in our fiber probes is analyzed numerically and an optimization strategy is presented.

\section{Brillouin Scattering}

In Brillouin scattering, an incoming photon (at the angular frequency $\omega_{i}$ and the wave vector $\mathbf{k}_{i}$ ) interacts with a traveling thermal phonon $(\Omega, \mathbf{K})$, resulting in the photon being scattered at an angle $\Theta=\angle\left(\mathbf{k}_{i}, \mathbf{k}_{s}\right)$ (Fig. 1) and with a Doppler frequency shift equal to the phonon frequency, i.e., $\omega_{s}=$ $\omega_{i} \pm \Omega$. The sign \pm corresponds to creation or annihilation of a phonon in the scattering event. Energy and momentum conservation laws lead to the relationship for the frequency shift $\Omega=$ $\frac{2 n v}{\lambda} \sin (\Theta / 2)$, where $n$ is the refractive index of the scattering medium, $v$ is the acoustic speed and $\lambda$ is the optical wavelength (in vacuum).

The scattering angle $\Theta$ is usually determined by the geometry of the experiment, and in this work we examine only situations when the scattering angle is $180^{\circ}$ or backscattering geometry. In this situation, the frequency shift is simply $\Omega=\frac{2 n v}{\lambda}$. We can

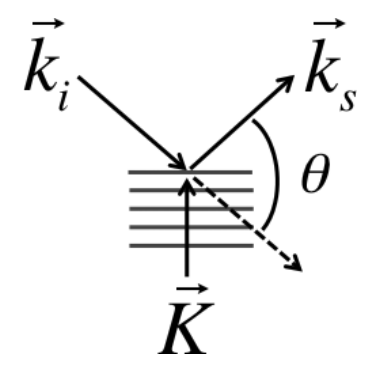

Fig. 1. Schematic of the inelastic Brillouin scattering process. 
immediately see that the Brillouin frequency shift $\Omega$ is proportional to the acoustic speed $v$, that in turn is a function of the longitudinal elastic modulus $M=\rho v^{2}$, with $\rho$ being the material density. In general, different acoustic modes propagate with different velocities. However, in the backscattering geometry $\left(\Theta=180^{\circ}\right)$ and in isotropic media, only longitudinal acoustic waves can be probed, resulting in a single Brillouin doublet arising in the spectrum of scattered light.

Biological tissues and cells generally have high liquid content $(>70 \%)$ and Poisson ratios $\eta \approx 0.5$ and are therefore very different in their mechanical properties compared to solids. The mechanical response of biomaterials and tissues can be approximately described by visco-elastic theory, according to which the longitudinal modulus becomes a complex number $M=M^{\prime}+i M^{\prime \prime}$. In this description the acoustic velocity is related to the real part of $M, v=\sqrt{M^{\prime} / \rho}$, whereas the width of the Brillouin peak $\Delta=\frac{\Omega M^{\prime \prime}}{\rho v^{2}}$ is associated with the acoustic damping and is proportional to the imaginary part of the modulus. The relation between the Brillouin frequency shift $\Omega$ and the more conventional mechanical parameters such as Young's modulus and shear modulus in biological tissues remains the subject of discussion. ${ }^{19,20}$ That is, however, beyond the scope of this paper.

\section{Experimental Methods}

Three optical configurations as shown in Fig. 2 were used to record Brillouin spectra of liquid samples: two fibre-probe arrangements, and for reference purposes, a bulk-optic arrangement. Figure 2(a) is a bulk-optic confocal arrangement as previously reported. ${ }^{13}$ A frequency-doubled, fiber-coupled Nd: $\mathrm{YVO}_{4}$ diode-pumped solid-state laser (CNI, MSLFN-671), operating at $\lambda=671 \mathrm{~nm}$ was used to probe the liquid. The spectrometer comprised an interference filter, a VIPA etalon (LightMachinery) with a free spectral range of $30 \mathrm{GHz}$ and a EMCCD camera (Andor iXon DU888). The laser output was collimated into an $8 \mathrm{~mm}$ diameter beam to fill the back focal plane of a $20 \times$ microscope objective (Olympus, $\mathrm{NA}=0.5$ ). The quarter-wave plate inserted just before the objective was used to transform the laser linear polarization into a circular polarization. Scattered light was collected by the same objective, separated from the incident light by the polarization beam-splitter and coupled into a single mode fiber (SMF), which also acted as a confocal pinhole. The final imaging resolution of this setup was calculated to be $1 \times 1 \times 5 \mu \mathrm{m}$.

Figure 2(b) is a single-fiber probe arrangement. The light from the laser was passed through a 50/50 fiber coupler (C), one output port of which was connected to a fiber ferrule and a graded-index lens (Thorlabs GRIN2906). The ferrule and the fiber lens both had diameter of $1.8 \mathrm{~mm}$, were aligned along the optical axis, brought in contact and glued together. The length of GRIN lens was chosen to be 0.29 of its pitch, resulting in the laser beam being focused at a distance of $1.35 \mathrm{~mm}$ after the lens. The diameter of the focal spot was measured to be approximately $3 \mu \mathrm{m}$, corresponding to the imaging resolution of $3 \times 3 \times 10 \mu \mathrm{m}$ for this system. The scattered light was collected by the same GRIN lens, coupled back to the SMF through the same fiber ferrule, and redirected to the spectroscope by

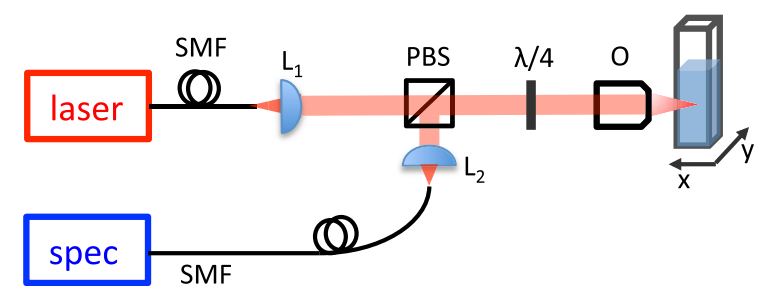

(a)

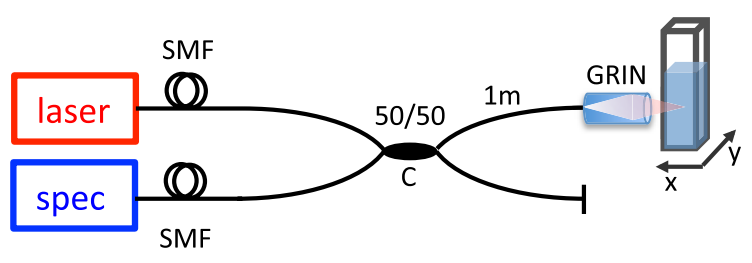

(b)

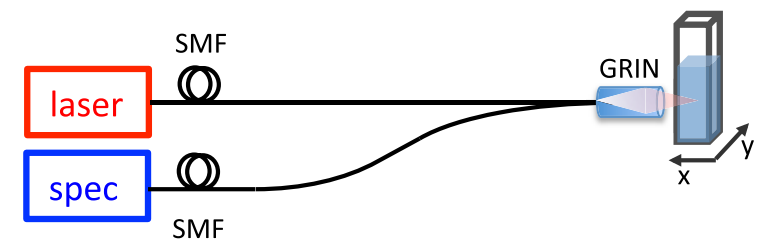

(c)

Fig. 2. Experimental setups: (a) - bulk-optic confocal microscope, (b) - single-fiber probe, (c) - dual-fiber probe. (Key: spec - spectrometer, $\mathrm{L}_{1}, \mathrm{~L}_{2}$ - aspherical lenses, $\mathrm{O}$ microscope objective, PBS - polarization beam-splitter, SMF — single-mode fiber, $\lambda / 4$ - quarter-wave plate, GRIN graded index fiber lens, $\mathrm{C}$ - fiber-coupler.) 
the fiber coupler. Thus, the measurement system $B$ presents the direct analog of the bulk-optical system A and, as A, has a confocal nature.

Figure 2(c) is a dual-fiber arrangement in which two optical fibers brought in contact with each other side-by-side, aligned symmetrically with the center of the GRIN lens and placed at the focal distance from the lens in a v-grove. In this instance, no fiber coupler was used: the light from the laser was sent directly to the sample through the GRIN lens, and the scattered signal was collected by the second fiber and directed towards the spectrometer.

\section{Measurement Results}

Samples of water, ethanol and glycerol were measured using the two fiber setups [Figs. 2(b) and 2(c)]. For reference purposes, we first used the confocal microscope arrangement [Fig. 2(a)] to record the spectrum for distilled water. The Brillouin frequency shift in water is well-documented as water is often used in Brillouin imaging experiments for calibration purposes. The central elastic (Rayleigh) peak and the Brillouin doublet of water are clearly visible in Fig. 3(a). The curve, shown with blue crosses, represents averaged results over 20 measurements, with each measurement taken using a $1 \mathrm{~s}$ acquisition time on the camera. The Brillouin peaks were fitted with a Lorentzian lineshape (in agreement with the mechanical oscillator model), to obtain a measured Brillouin frequency shift of $\Omega_{w}=5.81 \pm 0.01 \mathrm{GHz}$. This frequency corresponds to an acoustic velocity of $1465 \pm 2.5 \mathrm{~ms}^{-1}$ (taking the refractive index of water as $n_{w}=1.332$ ), the result expected for distilled water at $20^{\circ} \mathrm{C}$.

Next, we tested the single-fiber Brillouin probe arrangement [Fig. 2(b)] with three types of liquids: water, ethanol and glycerol. The results of this test are presented in Fig. 3(b). Since all three measurements overlap, we use different symbols for water (blue cross) and glycerol (yellow open circle), and a solid red line for the measurement of ethanol. In all three measurements, the Brillouin signal is shifted by $5.07 \pm 0.01 \mathrm{GHz}$ from the central Rayleigh peak. It is known that the Brillouin frequencies of the three liquids are, in fact, substantially different. ${ }^{21,22}$

The explanation of the measurement results becomes clear if we recall that the probe light, before being focused into the sample, passes through a $1 \mathrm{~m}$-long piece of a single-mode optical fiber (from

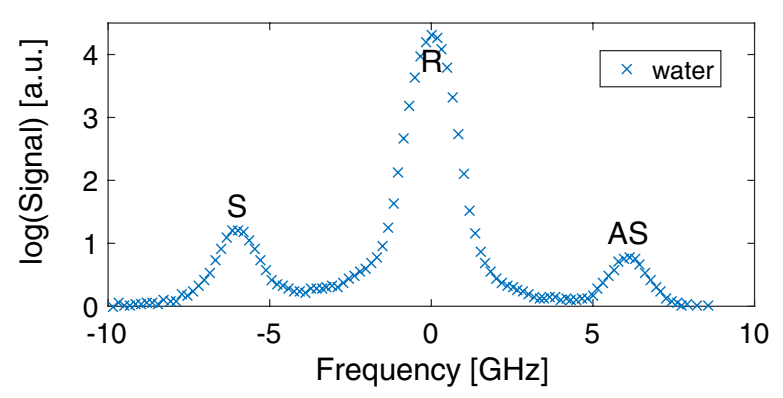

(a)

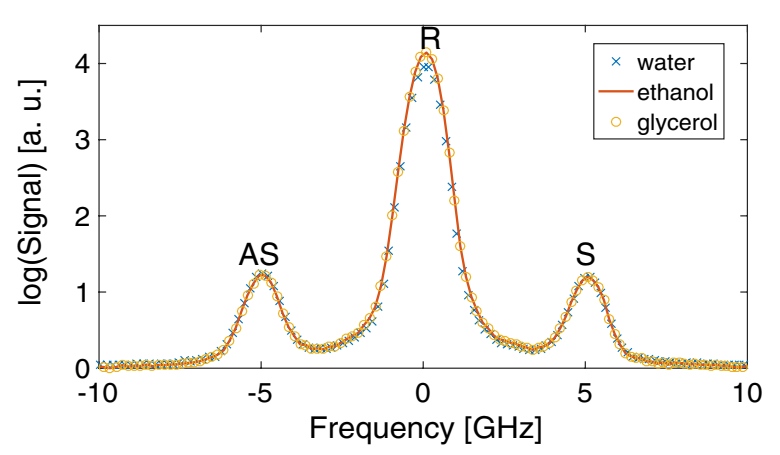

(b)

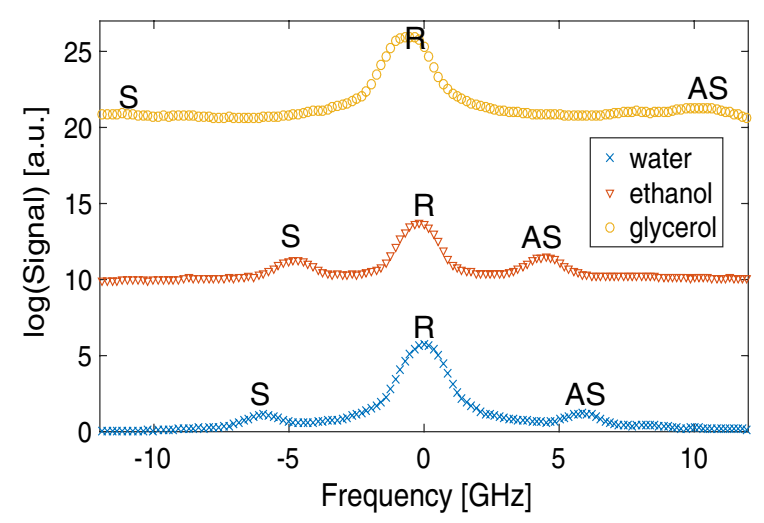

(c)

Fig. 3. (Color online) Brillouin spectra acquired using the three optical setups of Figs. 2(a)-2(c) as per Fig. (2) for water, ethanol and glycerol. The spectra all comprise a central elastic peak (R) and a Brillouin doublet, Stokes (S) and AS peaks, respectively. All three measurements in B are overlapping; hence it is likely that the Brillouin signal originates not from the liquid samples, but from the fiber itself. Note that the spectra in $\mathrm{C}$ are separated vertically by 10 units for better visibility.

the fiber coupler). In the fiber core, the light undergoes spontaneous Brillouin scattering in the backward direction, that is combined with the signal scattered by the sample. In fact, the amount of light scattered inside the fiber is expected to be several orders of magnitude larger compared to the 
light scattered inside the liquid sample. The ratio of these two signals scales roughly with the ratio of the scattering volumes in the fiber and liquid, respectively, so $V_{f} / V_{l} \approx L_{f} / L_{l}=1 \mathrm{~m} / 10 \mu \mathrm{m}=10^{5}$, since the mode field diameter of the single-mode fiber and the GRIN lens' focal spot diameter are approximately the same $(d=3 \mu \mathrm{m})$. The difference between the scattering efficiency in liquids and solids is not taken into account in this calculation, although the scattering efficiency of solids is typically higher. Thus, the factor $10^{5}$ is likely to be a conservative estimate for the ratio of light scattering in a glass fiber versus liquid samples.

This indicates that the side-lobes in Fig. 3(b) correspond to Brillouin scattering from the fiber rather than the liquid samples, which is expected to dominate the spectral measurements based on our estimates. Indeed, if we consider that the Brillouin signal to the right of the Rayleigh peak in Fig. 3(b) is, in fact, the Stokes signal (this is possible if the frequency shift is larger than half of the free spectral range of the VIPA), and that to the left of the Rayleigh peak is the AS signal, then we arrive at $\Omega_{\text {glass }}=24.93 \pm 0.01 \mathrm{GHz}$, in close agreement with previously reported value for Brillouin frequency shift in fused silica glass, corrected for the wavelength of the probing laser. ${ }^{23}$

The results of using a single-fiber Brillouin probe demonstrate that the scattering by the fiber itself presents a significant challenge in Brillouin endoscopy. This situation closely resembles the problem encountered in the field of Raman endoscopy. ${ }^{24,25}$ Over the last 20 years of technological and scientific development, the field of Raman endoscopy has reached relative maturity with clinical applications being possible today. ${ }^{18}$ The process of designing a sensitive and efficient Raman fiber probe, however, has to overcome the same hurdle, i.e., the signal coming from the sample has to be isolated from the fiber background. The main difference between Brillouin and Raman light scattering is their spectral properties, namely the Brillouin signals being narrow-band (tens of $\mathrm{MHz}$ ) and shifted by only 1-30 GHz from the elastic peak (a narrow band that renders traditional filters, e.g., dielectric filters useless), which imposes additional, non-trivial challenges in overcoming this hurdle. On the other hand, unlike Raman scattering, spontaneous Brillouin scattering in optical fibers only occurs in backward direction, since forward scattering does not satisfy the phase-matching condition between

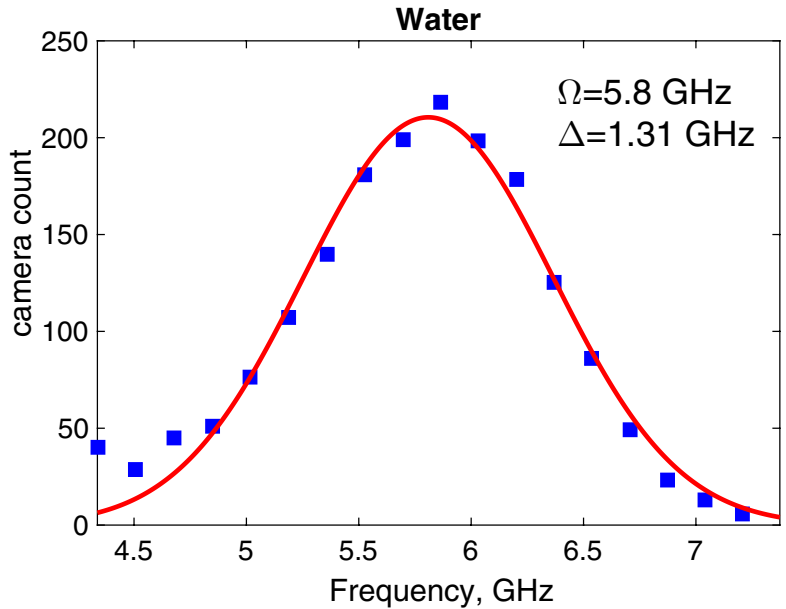

(a)

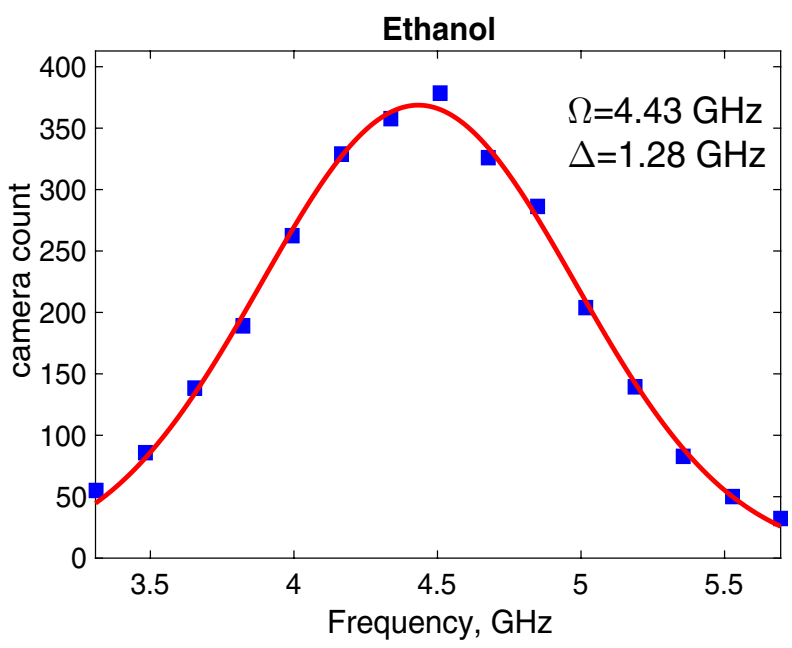

(b)

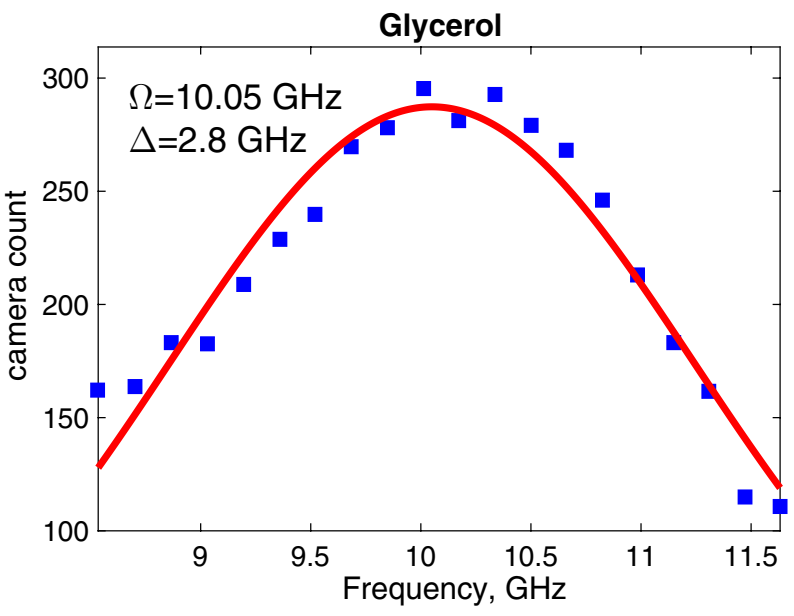

(c)

Fig. 4. Brillouin spectral peaks recorded using the dual-fiber setup [Fig. 2(c)] for water, ethanol and glycerol (markers) fitted with Lorentzian lineshapes (solid lines.) 
optical and acoustic waves. This fact becomes very important in construction of the Brillouin endoscope in a backscattering geometry using multiple fibers. Choosing separate fiber channels for the probe and return beam prevents back-scattering of the probe in the fiber from reaching the spectrometer.

The results of using this dual-fiber approach [Fig. 2(c)] are presented in Fig. 3(c). In contrast with the spectra obtained with the single-fiber arrangement, it can be seen that the side-peaks are different for each sample. After fitting the measurement data with Lorentzian curves (Fig. 4), we arrive at the following Brillouin frequency shifts $\Omega_{w}=5.8 \pm 0.01 \mathrm{GHz}, \Omega_{\text {eth }}=4.43 \pm 0.01 \mathrm{GHz}$ and $\Omega_{\text {glyc }}=10.05 \pm 0.01 \mathrm{GHz}$ in water, ethanol and glycerol, respectively. These are consistent with the values of Brillouin frequency shifts reported in literature. ${ }^{21,22}$

\section{Numerical Modeling of Light Propagation in Fiber Endoscopes}

Although the single-fiber arrangement of Fig. 2(b) is confocal in nature, this is no-longer the case for the dual-fiber arrangement of Fig. 2(c), since the two fiber cores are separated laterally by $250 \mu \mathrm{m}$, the diameter of the fiber cladding. The apertures of the illumination and collection fibers are located $125 \mu \mathrm{m}$ away from the center of the GRIN lens, which can lead to optical aberrations and poor overlap between illumination and collection volumes. To understand the effect of this fiber core separation on the collection efficiency of the scattered light, we approximate the light exiting from the optical fiber by a Gaussian beam with the full width at half maximum of $3 \mu \mathrm{m}$ (equal to the mode field diameter of the SMF) and use the beam propagation method to study numerically the light propagation in our fiber probes. ${ }^{26}$

Since the fiber and the lens have cylindrical symmetry, we restrict our model to the twodimensional plane $(X, Z)$ with $z$-direction being along the optical axis of the lens. The GRIN lens (Thorlabs) is modeled as a variable refractive index profile

$$
n(x, z)= \begin{cases}1 & \text { if } z<l_{0} \\ n_{0}\left(1-A x^{2} / 2\right) & \text { if } l_{0} \leq z \leq L+l_{0} \\ 1 & \text { if } z>L+l_{0}\end{cases}
$$

Here, $l_{0}=1.35 \mathrm{~mm}$ is the focal distance of the lens, $L=5.38 \mathrm{~mm}$ is the lens length, $n_{0}=1.607$ is the maximum refractive index of the lens and $A=$ 0.1149 is the coefficient of the refractive index curvature.

Results of the beam propagation modeling are shown in Fig. 5. Figure 5(a) shows a single Gaussian beam, placed at the focal plane of the GRIN lens, and traveling through the lens. As expected, the beam is focused behind the lens at the focal distance and forms a good image of the initial beam. This situation corresponds to the single-fiber probe setup.

Figure 5(b) shows two Gaussian beams, placed in the focal plane in front of the lens and separated vertically by $250 \mu \mathrm{m}$. This corresponds to the dualfiber arrangement. In the experiment, only one fiber delivers light, whereas the second fiber collects the scattered signal. Since the system is time-reversible, it does not matter if the source is placed from the left or the right side of the lens. Thus, by putting both sources at the apertures of optical fibers, we can trace the beams trajectories and analyze their overlap at the image plane. The collection efficiency is dependent on a good overlap between the illumination and collection beams at the scattering sample. As is clear from Fig. 5(b), the illumination and collection volumes do not overlap significantly at the sample plane, meaning that negligible amount of light scattered by the sample can be coupled back into the collection fiber. Although we have measured Brillouin signals in transparent and homogeneous liquids (water, ethanol and glycerol) using a dual-fiber probe, the poor overlap between illumination and collection volumes, characteristic for this endoscope design, makes its use in conditions of highly scattering biological tissues problematic.

Next, we explored different separation distances between the fibers and the fiber tilt angles in an attempt to seek an optimal scenario, where the images of the two Gaussian beams overlap. Figure 5(c) illustrates one such configuration, in which the fiber cores are separated by $50 \mu \mathrm{m}$ and each core is tilted by $6^{\circ}$ away from the optical axis. One can see that although the images of the beams show significant aberrations they do in fact overlap, which suggests higher collection efficiency for this probe design compared to the previous one. The tilt angle and the core separation conditions can be realized through polishing of separate single-mode 


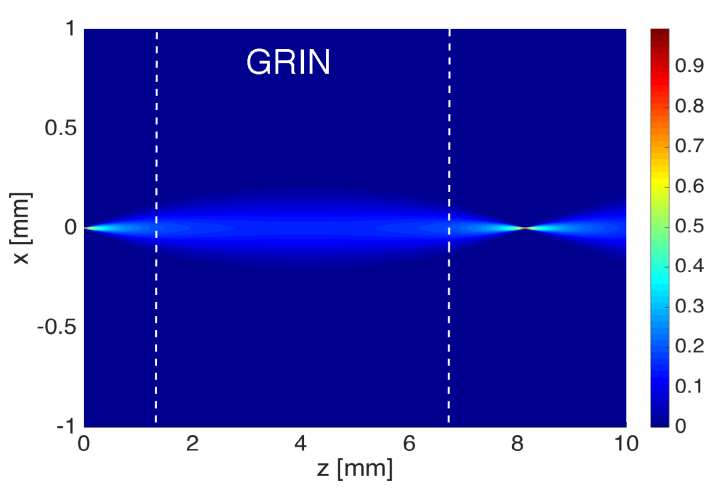

(a)

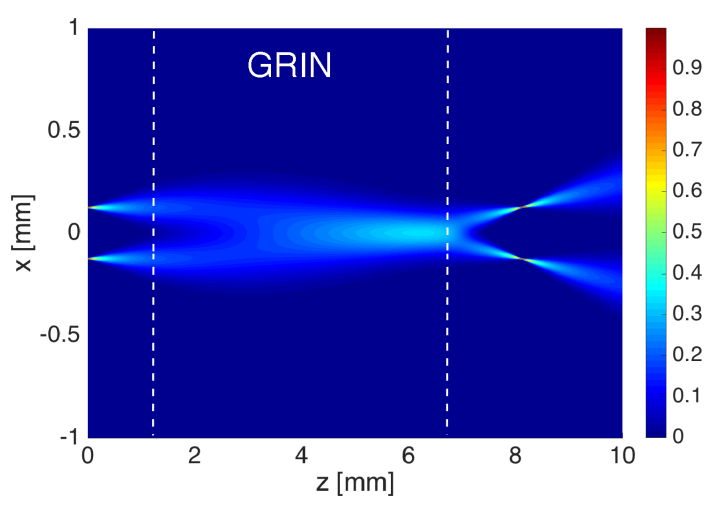

(b)

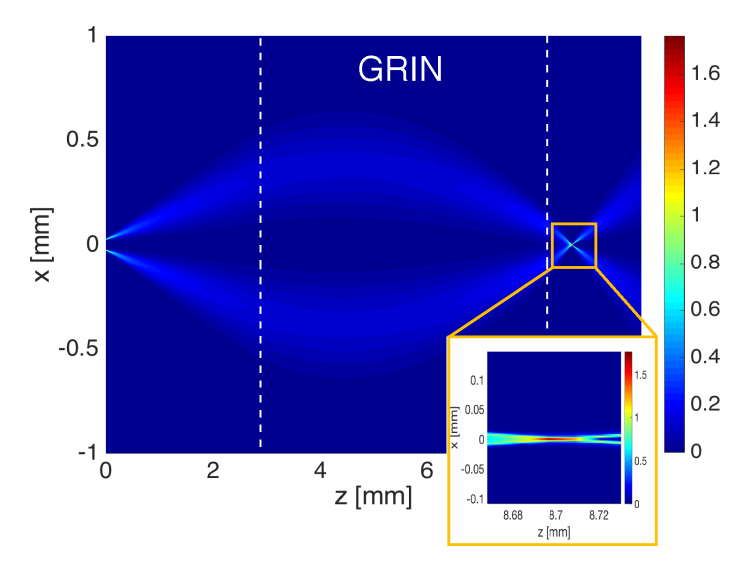

(c)

Fig. 5. Two-dimensional propagation modeling of Gaussian beams in a GRIN lens. (a) Single beam placed at the focal plane. (b) Two beams placed at the focal plane of the lens, but each shifted by $125 \mu \mathrm{m}$ vertically from the optical axis. (c) Two beams shifted from the focal plane and tilted by a $6^{\circ}$ angle. The beams overlap area is $5 \mu \mathrm{m} \times 20 \mu \mathrm{m}$. White dotted lines indicate the ends of the GRIN lens.

optical fibers, or possibly through fabrication of a dual-core fiber, which facet is polished to a conical shape with the required angle. An alternative solution could be a complex fiber lens, fabricated by the direct laser writing method on the tip of a multicore optical fiber that would enable light focusing in a single aberration-free spot. ${ }^{27}$

\section{Discussion and Conclusions}

Based on our experiments using a single-fiber and a dual-fiber Brillouin probes, we have concluded that the former design is not suitable due to significant backscattering inside the fiber, whereas the latter is suitable for Brillouin endoscopy after improvements in the collection efficiency. Our simulation results suggest that a simple arrangement of a two optical fibers and a GRIN lens does not have the desired overlap between the illumination and the collection volumes at the image plane of the lens. Improvements on the dual-probe design, namely reduction of the core separation and the tilt, may help to increase the collection efficiency by allowing for a better optical overlap between the illumination and collections volumes.

It is worth noting that, despite the poor collection efficiency, we were able to obtain clear Brillouin signals from the three liquids using the dual-fiber probe. In fact, the signal-to-noise ratio (SNR) in the measured spectra was similar to that using a conventional arrangement with a confocal microscope [setup illustrated in Fig. 2(a) and the measurement result shown in Fig. 3(a)]. This might seem surprising at first because of the much lower collection efficiency, however it should be noted that other factors affect the SNR, such as stray reflections from optical elements and Rayleigh scattering. Although, we have utilized polarization rotation and filtering in the setup 2(A) in order to reduce amount of stray light being sent to the spectrometer, the finite extinction ratio of the polarization beam splitter and imperfections of the alignment could explain a small fraction of stray light being added to the Brillouin signal. In the dual-fiber geometry, however, the chance of stray light reaching the detector is negligible since the illumination and the collection paths are completely separated. Also, the lower collection efficiency also reduces the amount of Rayleigh light being collected and sent to the spectrometer.

In summary, we have demonstrated Brillouin microspectroscopy measurements of liquids (water, ethanol and glycerol) using fiber-integrated BI system. We have designed a single and a dual-fiber probes using off-the-shelf components and demonstrated Brillouin spectroscopy measurements using 
the dual-fiber probe. The main challenge of the Brillouin endoscopy using optical fibers has been identified, namely the detrimental effect of the inelastic scattering in fibers. We believe that this work represents an important first step towards simplified, fiber-integrated BI setups, that in the future could find applications in biomedical research and clinical trials. The next step in the development of fiber probes for Brillouin endoscopy could be the optimization of the dual-fiber design in order to provide better coupling efficiency of the scattered light.

\section{Acknowledgments}

Dr. Kabakova is thankful for the financial support provided through Imperial College Research Fellowship Scheme (G53037).

\section{References}

1. K. J. Koski, J. L. Yarger, "Brillouin imaging," Appl. Phys. Lett. 87, 061903 (2005).

2. L. Brillouin, Ann. Phys. 17, 873-875 (1922).

3. G. Scarcelli, S. H. Yun, "Confocal Brillouin microscopy for three-dimensional mechanical imaging," Nat. Photon. 2, 39-43 (2008).

4. G. Scarcelli, S. H. Yun "In vivo Brillouin optical microscopy of the human eye," Opt. Express 20, 9197-9202 (2012).

5. G. Antonacci, R. M. Pedrigi, A. Kondiboyina, V. V. Mehta, R. de Silva, C. Paterson, R. Krams, P. Török, "Quantification of plaque stiffness by Brillouin microscopy in experimental thin cap fibroatheroma," J. R. Soc. Interface 12, 20150843 (2015).

6. I. L. Fabelinskii, Molecular Scattering of Light (Springer, New York, 1968).

7. D. S. Bedborough, D. A. Jackson, "Brillouin scattering study of gelatin gel using a double passed Fabry-Perot spectrometer," Polymer 17, 573-576 (1976).

8. R. Harley, D. James, A. Miller, J. W. White, "Phonons and the elastic moduli of collagen and muscle," Nature 267, 285-287 (1977).

9. S. Cusack, A. Miller, "Determination of the elastic constants of collagen by Brillouin light scattering," J. Mol. Biol. 135, 39-51 (1979).

10. F. Palombo, C. P. Winlove, R. S. Edginton, E. Green, N. Stone, S. Caponi, M. Madami, D. Fioretto, "Biomechanics of fibrous proteins of the extracellular matrix studied by Brillouin scattering," J. R. Soc. Interface 11, 20140739 (2014).
11. J. R. Sandercock, "Simple stabilization scheme for maintenance of mirror alignment in a scanning February-Perot interferometer," J. Phys. E, J. Sci. Instrum. 9, 566-569 (1976).

12. M. Shirasaki, "Virtual image phase array," Fujitsu Sci. Tech. J. 35, 113-125 (1999).

13. G. Lepert, R. M. Gouveia, C. J. Connon, C. Paterson, "Assessing corneal biomechanics with Brillouin spectro-microscopy," Faraday Discuss. 187, 414-428 (2016).

14. G. Antonacci, G. Lepert, C. Paterson, P. Török, "Elastic suppression in Brillouin imaging by destructive interference," Appl. Phys. Lett. 107, 061102 (2015).

15. A. J. Traverso, J. V. Thompson, Z. A. Steelman, Z. Meng, M. O. Scully, V. V. Yakovlev, "Dual Raman-Brillouin Microscope for Chemical and Mechanical Characterization and Imaging," Anal. Chem. 87, 7519-7523 (2015).

16. K. Berghaus, J. Zhang, S. H. Yun, G. Scarcelli, "High-finesse sub-GHz-resolution spectrometer employing VIPA etalons of different dispersion," Opt. Lett. 40, 4436-4439 (2015).

17. J. Zhang, A. Fiore, S. H. Yun, H. Kim, G. Scarcelli, "Line-scanning Brillouin microscopy for rapid noninvasive mechanical imaging," Sci. Rep. 6, 35398 (2016).

18. J. C. C. Day, R. Bennett, B. Smith, C. Kendall, J. Hutchings, G. M. Meaden, C. Born, S. Yu, N. Stone, "A miniature confocal Raman probe for endoscopic use," Phys. Med. Biol. 54, 7077-7087 (2009).

19. G. Scarcelli, P. Kim, S. H. Yun, "In Vivo Measurement of Age-Related Stiffening in the Crystalline Lens by Brillouin Optical Microscopy," Biophys. J. 101, 1539-1545 (2011).

20. G. Scarcelli, W. J. Polacheck, H. T. Nia, K. Patel, A. J. Grodzinsky, R. D. Kamm, S. H. Yun, "Noncontact three-dimensional mapping of intracellular hydromechanical properties by Brillouin microscopy," Nat. Methods 12, 1132-1134 (2015).

21. D. H. Rank, E. M. Kiess, Uwe Fink, T. A. Wiggins, "Brillouin spectra of liquids Using HeNe Laser," J. Opt. Soc. Am. 55, 925-927 (1965).

22. M. S. Jeong, J. H. Ko, Y. H. Ko, K. J. Kim, "Highpressure acoustic properties of glycerol studied by Brillouin spectroscopy," Phys. B, Cond. Matt. 478, 27-30 (2015).

23. H. Gu, H. Dong, G. Zhang, Y. Dong, J. He, "Dependence of Brillouin frequency shift on radial and axial strain in silica optical fibres," Appl. Opt. 32, 7864-7868 (2012).

24. I. Latka, S. Dochow, C. Krafft, B. Dietzek, J. Popp, "Fiber optic probes for linear and nonlinear Raman applications current trends and future 
development," Laser Photon. Rev. 17, 698-731 (2013).

25. S. Brustlein, P. Berto, R. Hostein, P. Ferrand, C. Billaudeau, D. Marguet, A. Muir, J. Knight, H. Rigneault, "Double-clad hollow core photonic crystal fiber for coherent Raman endoscope," Opt. Express 19, 12562-12568 (2011).
26. M. D. Feit, J. A. Fleck, "Light propagation in graded-index optical fibers," Appl. Opt. 17, 39903998 (1978).

27. T. Gissibl, S. Thiele, A. Herkommer, H. Giessen, "Two-photon direct laser writing of ultracompact multi-lens objectives," Nat. Photon. 10, 554-560 (2016). 